\title{
K-Rb Fermi-Bose mixtures: Vortex states and sag
}

\author{
D. M. Jezek \\ Departamento de Física, Facultad de Ciencias Exactas y Naturales, Universidad de Buenos Aires, RA-1428 Buenos Aires, Argentina \\ and Consejo Nacional de Investigaciones Científicas y Técnicas, Argentina \\ M. Barranco, M. Guilleumas, R. Mayol, and M. Pi \\ Departament d'Estructura i Constituents de la Matèria, Facultat de Física, Universitat de Barcelona, E-08028 Barcelona, Spain
}

(Received 3 May 2004; published 29 October 2004)

\begin{abstract}
We study a confined mixture of bosons and fermions in the quantal degeneracy regime with attractive boson-fermion interaction. We discuss the effect that the presence of vortical states and the displacement of the trapping potentials may have on mixtures near collapse, and investigate the phase stability diagram of the K-Rb mixture in the mean-field approximation supposing in one case that the trapping potentials felt by bosons and fermions are shifted from each other, as it happens in the presence of a gravitational sag, and in another case, assuming that the Bose condensate sustains a vortex state. In both cases, we have obtained an analytical expression for the fermion effective potential when the Bose condensate is in the Thomas-Fermi regime, that can be used to determine the maxima of the Fermionic density. We have numerically checked that the values one obtains for the location of these maxima using the analytical formulas remain valid up to the critical boson and fermion numbers, above which the mixture collapses.
\end{abstract}

DOI: $10.1103 /$ PhysRevA.70.043630

PACS number(s): 03.75.Mn, 03.75.Lm, 32.80.Pj

\section{INTRODUCTION}

Recent experiments on degenerate Fermi-Bose mixtures have opened the possibility of studying in a direct way the effect of quantum statistics in Bose-Einstein condensates (BEC's). In practice, the direct evaporative cooling techniques used to obtain BEC's are not applicable in a Fermi gas, as the Pauli exclusion principle forbids $s$-wave collisions between fermions. This difficulty has been overcome using a gas of bosons as a coolant, which has given further relevance to the study of these mixtures.

One of the systems studied in recent experiments is the ${ }^{6} \mathrm{Li}-{ }^{7} \mathrm{Li}$ mixture $[1,2]$. It is characterized by having a positive boson-fermion scattering length an order of magnitude larger than the boson-boson one [2]. Due to this repulsive bosonfermion interaction, the system does not exhibit a large overlap between the two species [3]. Moreover, this mixture may undergo a two-component separation $[3,4]$. Both facts conspire against having the fermions well inside the boson cloud, which is desirable to obtain an efficient sympathetic cooling. In contrast, this desirable overlap can be achieved in ${ }^{40} \mathrm{~K}-{ }^{87} \mathrm{Rb}$ mixtures due to the large attractive boson-fermion interaction. This mixture has been recently obtained [5-7], and it has been shown that if the particle numbers are above some critical values the system collapses [7].

From a theoretical point of view, a fairly amount of work has been devoted to the study of boson-fermion mixtures $[3,8,9]$. In particular, a systematic study of the structure of binary mixtures has been performed in Ref. [3], where all possible sign combinations of scattering lengths between boson-boson and boson-fermion $s$-wave interactions have been discussed. The purpose of our work is, first, to present an analysis of the location of the minima of the effective potential felt by fermions submitted to a large number of condensate bosons when the boson-boson interaction is repulsive, and second, to extend this study to systems with a large number of fermions with an attractive boson-fermion interaction.

An essential characteristic of superfluid systems is the occurrence of quantized vortices [10]. Quantized vortices in BEC's were produced experimentally by Matthews et al. [11], and have been studied since in detail (see Refs. [12-14] and references therein). It may thus be interesting to see what is the physical appearance of such vortices, arising in the Bosonic component, in the presence of a Fermionic cloud which is in the normal (nonsuperfluid) but quantum degenerate phase. This is another aim of our work, which bears some similarities with the description of quantized vortices in ${ }^{3} \mathrm{He}-{ }^{4} \mathrm{He}$ nanodroplets recently addressed [15].

This work is organized as follows. In Sec. II we describe the mean-field model we have used. In Sec. III we present an analysis of the effective potential felt by the fermions when they are inside a large condensate that exhibits a bosonboson repulsive interaction, and the number of fermions $\left(N_{F}\right)$ is much smaller than the number of bosons $\left(N_{B}\right)$. We shall consider the case in which the boson and fermion external confining potentials are displaced from each other, which may be caused by gravity for instance, and also when bosons are in a vortical state. In Sec. IV we present the results obtained by solving the mean-field coupled equations for different $N_{B}$ and $N_{F}$ values up to the critical values where collapse of the mixture occurs. A summary is given in Sec. V. Finally, in the Appendix we derive some expressions using a scaling transformation which, on the one hand, constitute a generalization of the virial theorem, and on the other hand are especially useful for testing the accuracy of the numerical procedure.

\section{MEAN-FIELD MODEL}

We consider a mixture of a Bose condensate (B) and a degenerate Fermi gas $(\mathrm{F})$ at zero temperature confined in an 
axially symmetric harmonic trap. Assuming that the minimum of the trapping potential felt by each species may be displaced in the $z$ direction in a value $d_{i}(i=\mathrm{B}, \mathrm{F})$, the confining potentials in cylindrical coordinates are

$$
V_{B}=\frac{1}{2} M_{B}\left[\omega_{r B}^{2} r^{2}+\omega_{z B}^{2}\left(z-d_{B}\right)^{2}\right]
$$

and

$$
V_{F}=\frac{1}{2} M_{F}\left[\omega_{r F}^{2} r^{2}+\omega_{z F}^{2}\left(z-d_{F}\right)^{2}\right],
$$

where $\omega_{r B}, \omega_{z B}$ and $\omega_{r F}, \omega_{z F}$ are the trapping radial (axial) angular frequencies for bosons and fermions, respectively. $M_{B}$ and $M_{F}$ are the corresponding masses.

Since the number of fermions we consider in the numerical calculations is fairly large, the Fermionic kinetic energy density can be written in the Thomas-Fermi-Weizsäcker (TFW) approximation as a function of the local fermion density $n_{F}$ and its gradients. For fully polarized spin- $1 / 2$ fermions, it reads

$$
\tau_{F}(\vec{r})=\frac{3}{5}\left(6 \pi^{2}\right)^{2 / 3} n_{F}^{5 / 3}+\beta \frac{\left(\nabla n_{F}\right)^{2}}{n_{F}}
$$

and the Fermionic kinetic energy is

$$
T_{F}=\frac{\hbar^{2}}{2 M_{F}} \int d \vec{r} \tau_{F}(\vec{r}) .
$$

The value of the $\beta$ coefficient in the Weizsäcker term is fixed to $1 / 18$. This term contributes little to the kinetic energy, and it is usually neglected [3]. However, its inclusion [16] has some advantages; see below. We refer the interested reader to Ref. [17] for a discussion on the accuracy of the TFW approximation (see also references therein).

Neglecting all $p$-wave interactions, the energy density functional for the boson-fermion mixture at zero temperature has the form

$$
\begin{aligned}
\mathcal{E}(\vec{r})= & \frac{\hbar^{2}}{2 M_{B}}|\nabla \Psi|^{2}+V_{B} n_{B}+\frac{1}{2} g_{B B} n_{B}^{2}+g_{B F} n_{F} n_{B}+\frac{\hbar^{2}}{2 M_{F}} \tau_{F} \\
& +V_{F} n_{F},
\end{aligned}
$$

where $n_{B}=|\Psi|^{2}$ is the local boson atomic density. The bosonboson and boson-fermion coupling constants $g_{B B}$ and $g_{B F}$ are written in terms of the $s$-wave scattering lengths $a_{B}$ and $a_{B F}$ as $g_{B B}=4 \pi a_{B} \hbar^{2} / M_{B}$ and $g_{B F}=4 \pi a_{B F} \hbar^{2} / M_{B F}$, respectively. We have defined $M_{B F} \equiv 2 M_{B} M_{F} /\left(M_{B}+M_{F}\right)$.

When bosons sustain a quantized vortex line along the $z$ axis, the condensate wave function can be written as $\Psi$ $=|\Psi| e^{i m \phi}$, where $m=1,2,3 \ldots$ is the circulation number, yielding for the kinetic energy density

$$
\frac{\hbar^{2}}{2 M_{B}}|\nabla \Psi|^{2}=\frac{\hbar^{2}}{2 M_{B}}(\nabla|\Psi|)^{2}+\frac{\hbar^{2} m^{2}}{2 M_{B}} \frac{n_{B}}{r^{2}} .
$$

We have considered only singly quantized vortices, that is $m=1$. If a vortex is present in the condensate, bosons flow around the vortex core with quantized circulation, which yields the centrifugal term in the kinetic energy. We assume that the Fermi component is not superfluid, and consider that it is in a stationary state. This situation could be achieved experimentally waiting enough time after the generation of the vortex in the condensate, to let the drag force between bosons and fermions to dissipate.

Variation of $\mathcal{E}$ with respect to $\Psi$ and $n_{F}$ keeping $N_{B}$ and $N_{F}$ fixed yields the following coupled Euler-Lagrange (EL) equations

$$
\left(-\frac{\hbar^{2} \nabla^{2}}{2 M_{B}}+V_{B}+\frac{\hbar^{2} m^{2}}{2 M_{B}} \frac{1}{r^{2}}+g_{B B} n_{B}+g_{B F} n_{F}\right) \Psi=\mu_{B} \Psi,
$$

$$
\begin{aligned}
& \frac{\hbar^{2}}{2 M_{F}}\left(\left(6 \pi^{2}\right)^{2 / 3} n_{F}^{5 / 3}+\beta \frac{\left(\nabla n_{F}\right)^{2}}{n_{F}}-2 \beta \Delta n_{F}\right)+V_{F} n_{F}+g_{B F} n_{B} n_{F} \\
& \quad=\mu_{F} n_{F},
\end{aligned}
$$

where $\mu_{B}$ and $\mu_{F}$ are the boson and fermion chemical potentials, respectively. Then, the ground state $(m=0)$ or a vortical state $(m=1)$ are obtained by solving the Gross-Pitaevskii (GP) equation for bosons [Eq. (7)] coupled to the ThomasFermi-Weizsäcker equation for fermions [Eq. (8)].

The inclusion of the Weizsäcker term in the energy density has the major advantage that it yields a EL equation for $n_{F}$ that is well behaved everywhere, avoiding the classical turning point problem when this term is neglected (ThomasFermi approximation). Moreover, solving Eq. (8) is not more complicated than solving the GP equation. This can be readily seen writing the latter in terms of $n_{B}$ :

$$
\begin{aligned}
& \frac{\hbar^{2}}{2 M_{B}}\left(\frac{1}{4} \frac{\left(\nabla n_{B}\right)^{2}}{n_{B}}-\frac{1}{2} \Delta n_{B}\right)+V_{B} n_{B}+\frac{\hbar^{2} m^{2}}{2 M_{B}} \frac{n_{B}}{r^{2}}+g_{B B} n_{B}^{2} \\
& \quad+g_{B F} n_{B} n_{F}=\mu_{B} n_{B},
\end{aligned}
$$

which is formally equivalent to Eq. (8). We have discretized these equations using nine-point formulas and have solved them on a two-dimensional (2D) $(r, z)$ mesh using a sufficiently large box. The results have been tested for different sizes of the spatial steps (we have mostly used $\Delta r=\Delta z$. $\sim 0.1 \mu \mathrm{m}$ ). We have employed the imaginary time method to find the solution of these coupled equations written as imaginary time diffusion equations [18]. After every imaginary time step, the densities are normalized to the corresponding particle numbers. To start the iteration procedure we have used positive random numbers to build both normalized densities. This avoids to introduce any bias in the final results. We have also checked that the solutions fulfill the generalized virial theorem deduced in the Appendix.

\section{EFFECTIVE FERMION POTENTIAL}

Before we present the numerical results obtained by solving Eqs. (8) and (9), it is useful to analyze the features of the effective potential felt by a small number of fermions in the presence of a boson condensate, paying special attention to the location of its minima. We are interested in studying the cases in which either the condensate hosts a vortex line or there exists a displacement between the minima of the boson and fermion external potentials. The latter situation is routinely met in the experiments-a gravitational sag in the $z$ direction-in which case the displacement is $g\left(1 / \omega_{z F}^{2}\right.$ $-1 / \omega_{z B}^{2}$ ), being $g$ the acceleration of gravity. 
An interesting issue is that the analytic expressions we obtain in this section for the location of the minimum of the effective potentials remain valid, as we will show in the next section, when the number of fermions and bosons are similar. These locations coincide with the positions of the maxima of the fermion density and are relevant because the collapse of the mixture originates precisely around them. This will be discussed in the next section.

The effective fermion potential has two main terms, the external potential and the mean-field term arising from the interaction with the boson condensate, which is proportional to $n_{B}$. We consider a large condensate in the Thomas-Fermi (TF) regime with positive scattering length and we assume that its density profile is not affected by the fermion presence. To obtain $n_{B}$ we may thus use Eq. (7) with $n_{F}=0$ and neglect the first kinetic energy term.

\section{A. Effect of a shift in the minimum of the external potentials}

For simplicity, we restrict ourselves to the vortex-free case $m=0$. Without loss of generality, the origin of coordinates can be fixed at the minimum of the boson trapping potential, and we will assume that the displacement is only in the $z$ direction. Thus the effective trapping potential experienced by the fermions is

$$
V_{d}^{\mathrm{eff}}=\frac{1}{2} M_{F}\left[\omega_{r F}^{2} r^{2}+\omega_{z F}^{2}\left(z-d_{z}\right)^{2}\right]+g_{B F} n_{B},
$$

where $d_{z}=d_{F}-d_{B}$ is the $z$ displacement between the trapping potential centers. Assuming that the density profile of the condensate is not affected by the interaction with fermions, the number of bosons is large, and that the interaction between bosons is repulsive $\left(g_{B B}>0\right)$, one obtains from Eq. (7) setting $m=0$ the boson density profile that corresponds to the uncoupled TF density

$$
\begin{aligned}
n_{B}= & \frac{1}{g_{B B}}\left(\mu_{B}-\frac{1}{2} M_{B}\left(\omega_{r B}^{2} r^{2}+\omega_{z B}^{2} z^{2}\right)\right) \Theta\left(\mu_{B}-\frac{1}{2} M_{B}\left(\omega_{r B}^{2} r^{2}\right.\right. \\
& \left.\left.+\omega_{z B}^{2} z^{2}\right)\right)
\end{aligned}
$$

with $\Theta(x)=1$ if $x>0$ and zero otherwise. The TF condensate boundary is given by the ellipsoid $\arg (\Theta)=0$. For an axially symmetric trap it yields the well-known TF radius of the condensate $R_{i}=\sqrt{2 \mu_{B} /\left(M_{B} \omega_{i B}^{2}\right)}$ with $i=r, z$ in the radial and axial direction, respectively. Replacing $n_{B}$ into Eq. (10) and defining the dimensionless parameter

$$
\gamma_{i} \equiv 1-\frac{a_{B F} M_{B}^{2} \omega_{i B}^{2}}{a_{B} M_{B F} M_{F} \omega_{i F}^{2}}
$$

with $i=r, z$, it follows that the effective fermion potential inside the Bose condensate-where $n_{B}$ [Eq. (11)] is positive-is

$$
\begin{aligned}
V_{d}^{\mathrm{eff}}= & \frac{1}{2} M_{F}\left[\gamma_{r} \omega_{r F}^{2} r^{2}+\gamma_{z} \omega_{z F}^{2}\left(z-d_{z} / \gamma_{z}\right)^{2}\right]+\frac{1}{2} M_{F} \omega_{z F}^{2} d_{z}^{2}(1 \\
& \left.-\gamma_{z}^{-1}\right)+\frac{g_{B F}}{g_{B B}} \mu_{B},
\end{aligned}
$$

whereas outside the condensate

$$
V_{d}^{\mathrm{eff}}=\frac{1}{2} M_{F}\left[\omega_{r F}^{2} r^{2}+\omega_{z F}^{2}\left(z-d_{z}\right)^{2}\right] .
$$

This effective potential can be viewed as having a renormalized frequency inside the boson condensate, a feature already discussed in Ref. [9] for a mixture with concentric external potentials. This model has been called the double-parabola model by Vichi et al. [9], and it has also been used by Capuzzi and Hernández [19]. However, in these works the possibility of a displacement between the minima of the potentials has not been considered.

The extremum of $V_{d}^{\text {eff }}$ inside the Bose condensate is attained at the point

$$
\vec{d}^{\prime}=\left(d_{x}^{\prime}, d_{y}^{\prime}, d_{z}^{\prime}\right)=\left(0,0, \gamma_{z}^{-1} d_{z}\right) .
$$

Note that if the shift between the external potentials is $\vec{d}$ $=\left(d_{x}, d_{y}, d_{z}\right)$, the effective potential has its extremum at $\vec{d}^{\prime}$ $=\left(\gamma_{x}^{-1} d_{x}, \gamma_{y}^{-1} d_{y}, \gamma_{z}^{-1} d_{z}\right)$. Depending on the signs of $\gamma_{i}, \vec{d}^{\prime}$ may correspond to a maximum, a minimum or a saddle point.

If the boson-fermion interaction is attractive $\left(g_{B F}<0\right)$ as for ${ }^{40} \mathrm{~K}-{ }^{87} \mathrm{Rb}$ mixtures, $\gamma_{i}>1$ and the point $\vec{d}^{\prime}$ is a minimum. Depending on the positions of $\vec{d}$ and $\vec{d}^{\prime}$ with respect to the ellipsoid $\arg (\Theta)=0$, with the argument of $\Theta$ taken from Eq. (11), there are three possibilities: (i) If $\vec{d}$ is inside this ellipsoid, fermions view only one minimum at $\vec{d}^{\prime}$; (ii) If $\vec{d}$ is outside and $\vec{d}^{\prime}$ is inside, there are two minima at $\vec{d}$ and $\vec{d}^{\prime}$; (iii) If both points are outside the ellipsoid, there is only one minimum at $\vec{d}$.

\section{B. Vortices}

We consider now the effect of a quantized boson vortex line in the fermion distribution, without gravitational sag. In this case, the effective fermion potential is

$$
V_{v}^{\mathrm{eff}}=V_{F}+g_{B F} n_{B}^{v}=\frac{1}{2} M_{F}\left(\omega_{r F}^{2} r^{2}+\omega_{z F}^{2} z^{2}\right)+g_{B F} n_{B}^{v},
$$

where $n_{B}^{v}$ is the boson density hosting a vortex line along the $z$ axis. This density is zero at $r=0$, and reaches its maximum value on a circle of radius $r_{0}$ around that axis. In the TF approximation, $n_{B}^{v}$ can be derived using Eq. (7) keeping the centrifugal term proportional to $m$, and may be approximately written as [12]

$$
n_{B}^{v}=\frac{1}{g_{B B}}\left(\mu_{B}-\frac{\hbar^{2} m^{2}}{2 M_{B}} \frac{1}{r^{2}}-V_{B}\right) \Theta\left(\mu_{B}-\frac{\hbar^{2} m^{2}}{2 M_{B}} \frac{1}{r^{2}}-V_{B}\right)
$$

with $r_{0}=\sqrt{\hbar m /\left(M_{B} \omega_{r B}\right)}$. Using $n_{B}^{v}$ we calculate the minimum of $V_{v}^{\text {eff }}$ inside the condensate, which is located at $z^{\prime}=0$ and

$$
r^{\prime}=\left(1-\gamma_{r}^{-1}\right)^{1 / 4} \sqrt{\frac{\hbar m}{M_{B} \omega_{r B}}}=\left(1-\gamma_{r}^{-1}\right)^{1 / 4} r_{0} .
$$

Thus the minimum is attained at a circle of radius $r^{\prime}$. We will see that for the ${ }^{40} \mathrm{~K}-{ }^{87} \mathrm{Rb}$ mixture $r^{\prime}$ is very close to the radius at which the boson density has its maximum. 


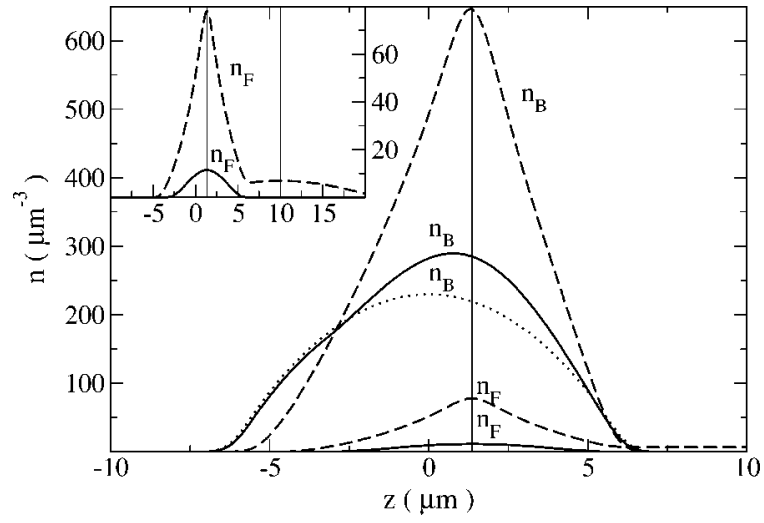

FIG. 1. Boson and fermion density profiles of ${ }^{40} \mathrm{~K}-{ }^{87} \mathrm{Rb}$ mixtures as a function of $z$ for a $d_{z}=10-\mu \mathrm{m}$ displacement. The different lines correspond to the profiles for $N_{B}=10^{5}$ and several $N_{F}$ values: $N_{F}=0$ (dotted line); $N_{F}=10^{3}$ (solid lines); $N_{F}=2.5 \times 10^{4}$ (dashed lines). The inset shows a magnified view of the fermion density distributions.

\section{NUMERICAL RESULTS}

The system under consideration is a confined ${ }^{40} \mathrm{~K}-{ }^{87} \mathrm{Rb}$ mixture. We have assumed spherically symmetric traps for bosons and fermions, with trap frequencies $\omega_{B}=2 \pi$ $\times 100 \mathrm{~Hz}$ and $\omega_{F}=\sqrt{M_{B} / M_{F}} \omega_{B}$. When a displacement $d_{z}$ is introduced between the minima of the external potentials, the mixture has only axial symmetry around the $z$ axis. We have numerically solved Eqs. (8) and (9) using the set of scattering lengths reported by Modugno et al. [7], namely $a_{B}$ $=98.98 a_{0}$, and $a_{B F}=-395 a_{0}$, being $a_{0}$ the Bohr radius. The dimensionless parameters introduced in Eq. (12) are $\gamma_{z}^{-1}$ $=\gamma_{r}^{-1}=0.136$, and $\left(1-\gamma_{r}^{-1}\right)^{1 / 4}=0.964$ [Eq. (18)].

We display in Fig. 1 several boson and fermion density profiles as a function of $z$, considering an arbitrary displacement $d_{z}=10 \mu \mathrm{m}$. They all correspond to $N_{B}=10^{5}$, but for three different fermion numbers: $N_{F}=0, N_{F}=10^{3}$, and $N_{F}$ $=2.5 \times 10^{4}$. The attractive boson-fermion interaction produces an enhancement of the density of both species in the overlap volume. However, this effect is reduced with respect to the concentric case due to $z$ displacement [7].

Using the TF approximation, the radius of the Bose condensate can be estimated as $R_{B}=\left(15 N_{B} a_{B} / a_{H O}\right)^{1 / 5} a_{H O}$, with $a_{H O}=\sqrt{\hbar / M_{B} \omega_{B}}$ being the oscillator length of the boson trap; this yields $R_{B} \sim 6 \mu \mathrm{m}$. Following the analysis performed in Sec. III A, and using $\gamma_{z}^{-1}=0.136$, we find that $d_{z}^{\prime}$ $=1.36 \mu \mathrm{m}<R_{B}$; thus within that model there are two minima in the effective fermion potential. One is at $\vec{d}=10(\mu \mathrm{m}) \hat{z}$, i.e., beyond the TF radius, and the other is inside the boson cloud, at $\vec{d}^{\prime}=1.36(\mu \mathrm{m}) \hat{z}$. It is interesting to note from Fig. 1 that in all cases, the maxima of the fermion density are precisely at $z=d_{z}^{\prime}$ and $z=d_{z}$, which we have displayed in the inset as vertical lines.

We thus see that for $N_{F} / N_{B}$ equal to $1 \%$ (solid line) or even $25 \%$ (dashed line), the maxima of the fermion density appear at the values obtained using the model of Sec. III A. This can be understood by solving Eqs. (7) and (8) with $m$ $=0$ (i.e., no vortex), without neglecting the effect of fermions in the GP equation as it was done in the simplified model of Sec. III, and assuming that all the gradient terms in both equations can be neglected. This assumption is justified for the number of bosons and fermions we have used: in the case of bosons, this is what the TF approximation is about, and in the case of fermions, the gradient term is a correction to the leading term proportional to $n_{F}^{5 / 3}$. Using Eq. (7), we obtain the following expression for $n_{B}$ :

$$
n_{B}=\frac{1}{g_{B B}}\left(\mu_{B}-V_{B}-g_{B F} n_{F}\right) .
$$

Replacing it into Eq. (8) and deriving the resulting expression with respect to $z$ we get

$$
\begin{gathered}
\frac{1}{3} \frac{\hbar^{2}}{M_{F}}\left(6 \pi^{2}\right)^{2 / 3} n_{F}^{-1 / 3} \frac{\partial n_{F}}{\partial z}-\frac{g_{B F}}{g_{B B}} M_{B} \omega_{z B}^{2} z+M_{F} \omega_{z F}^{2}\left(z-d_{z}\right) \\
-\frac{g_{B F}^{2}}{g_{B B}} \frac{\partial n_{F}}{\partial z}=0 .
\end{gathered}
$$

The extremum of the fermion density is found by setting $\partial n_{F} / \partial z=0$, with $n_{F} \neq 0$. After some algebra we find that the following equation has to be fulfilled:

$$
M_{F} \omega_{z F}^{2}\left(\gamma_{z} z-d_{z}\right)=0
$$

whose solution coincides precisely with the value $z=d_{z}^{\prime}$ we have found in the previous section.

Whereas the maximum of the fermion density is located at $z=d_{z}^{\prime}$ irrespective of the value of the dilution $\left(N_{F} / N_{B}\right)$, it can be seen from the numerical results that the maximum of the boson density moves from $z=0$ towards $z=d_{z}^{\prime}$ as $N_{F}$ increases. In fact, Fig. 1 shows that even for a rather small $N_{F}$ value $\left(N_{F}=10^{3}, N_{B}=10^{5}\right)$, the shape of the condensate differs from the parabolic-type profile yielded by the TF approximation for a fermion-free condensate, Eq. (11). It can be also appreciated in the inset that for a dilution of $N_{F} / N_{B}=25 \%$, a fairly large number of fermions remains without mixing (long tail in the fermion density profile outside the Bose condensate).

We plot in Fig. 2 several density profiles for configurations hosting a singly quantized vortex line in the case of no displacement between the trapping potentials. We have taken $N_{B}=10^{5}$, and have considered three different fermion numbers, $N_{F}=0,10^{3}$, and $1.5 \times 10^{4}$. When $N_{F}=0$, the maximum of the Bosonic density is located in the $z=0$ plane, in a circle of $r_{0}=1.13-\mu \mathrm{m}$ radius marked with a vertical line in the graph. Fermions are peaked around $r^{\prime}=0.96 r_{0}$, as we have obtained using the simplified model of Sec. III B with (1 $\left.-\gamma_{r}^{-1}\right)^{1 / 4}=0.964$.

It may be seen that in the presence of a quantized vortex, the position of the maximum of boson and fermion densities are very close irrespective of the dilution $N_{F} / N_{B}$. It is interesting to note that increasing $N_{F}$ at constant $N_{B}$, the fermion and boson density profiles become sharper due to their mutual attraction, eventually collapsing for a critical number of atoms. 


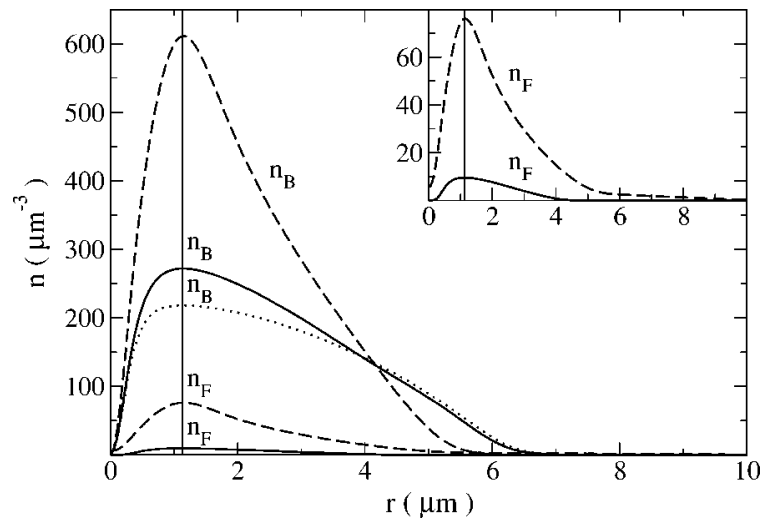

FIG. 2. Boson and fermion density profiles of ${ }^{40} \mathrm{~K}-{ }^{87} \mathrm{Rb}$ configurations hosting a vortex line with $m=1$ without displacement of the trapping potentials. In all cases, $N_{B}=10^{5}$. The different lines correspond to $N_{F}=0$ (dotted line); $N_{F}=10^{3}$ (solid lines); $N_{F}=1.5 \times 10^{4}$ (dashed lines). The inset shows a magnified view of the fermion density distributions.

Following a procedure analogous to that used before, we can justify the position of the maximum of the fermion density in the presence of a vortex in a TF condensate. From Eq. (7) we obtain

$$
n_{B}=\frac{1}{g_{B B}}\left(\mu_{B}-V_{B}-\frac{\hbar^{2} m^{2}}{2 M_{B}} \frac{1}{r^{2}}-g_{B F} n_{F}\right) .
$$

Replacing it into Eq. (8) and deriving the resulting expression with respect to $r$ we get

$$
\begin{aligned}
\frac{1}{3} \frac{\hbar^{2}}{M_{F}} & \left(6 \pi^{2}\right)^{2 / 3} n_{F}^{-1 / 3} \frac{\partial n_{F}}{\partial r}-\frac{g_{B F}}{g_{B B}} M_{B} \omega_{r B}^{2} r+M_{F} \omega_{r F}^{2} r+\frac{\hbar^{2} m^{2}}{M_{B}} \frac{1}{r^{3}} \\
& -\frac{g_{B F}^{2}}{g_{B B}} \frac{\partial n_{F}}{\partial r}=0 .
\end{aligned}
$$

Once again, the extremum in the Fermionic density is found by setting $\partial n_{F} / \partial r=0$ with $n_{F} \neq 0$, which means that the following equation has to be fulfilled:

$$
-\frac{g_{B F}}{g_{B B}} M_{B} \omega_{r B}^{2} r+M_{F} \omega_{r F}^{2} r+\frac{\hbar^{2} m^{2}}{M_{B}} \frac{1}{r^{3}}=0,
$$

whose solution again coincides with $r=r^{\prime}$ as found in the previous section [Eq. (18)].

Due to the attractive boson-fermion interaction, stable trapped ${ }^{40} \mathrm{~K}-{ }^{87} \mathrm{Rb}$ mixtures may only have a limited number of fermions and bosons. If the atom numbers increase above some critical values $N_{B}^{c}$ and $N_{F}^{c}$, an instability occurs [3]. It has been shown that the mean-field framework is able to reproduce the critical numbers for collapse [7]. We have calculated the stability diagram of the ${ }^{40} \mathrm{~K}-{ }^{87} \mathrm{Rb}$ mixture by solving the coupled mean-field equations (8) and (9) for different values of $N_{B}$ and $N_{F}$. In our study, the instability signature is the failure of the numerical iterative process. In particular, the instability onset appears as an indefinite growth of the maximum of the densities, which triggers the collapse.

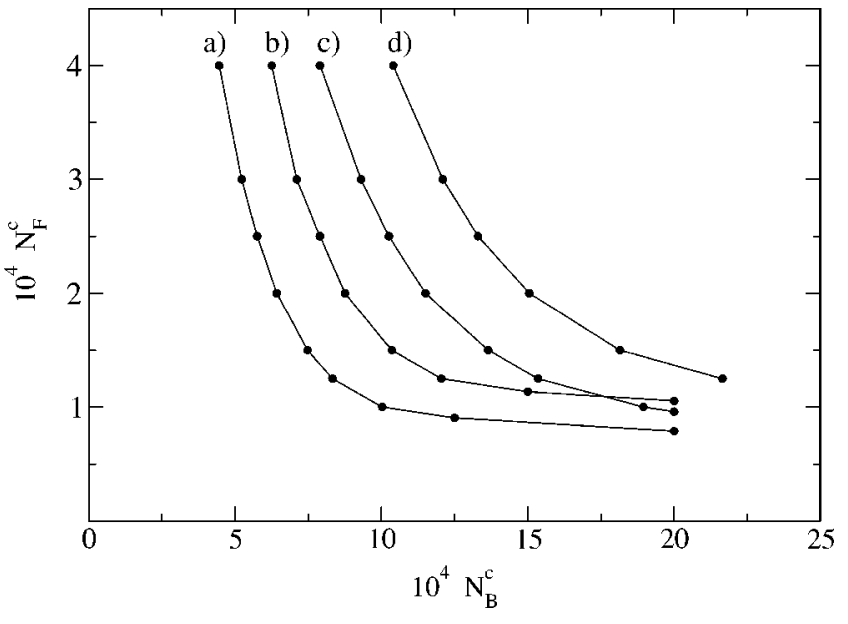

FIG. 3. Stability diagram of the ${ }^{40} \mathrm{~K}-{ }^{87} \mathrm{Rb}$ mixture as a function of the number of atoms. The dots are the prediction for the critical number of bosons and fermions. The lines have been drawn to guide the eye, and represent the critical instability lines that determine the boundary between the stable (left) and unstable (right) regions in four different cases: (a) vortex-free configurations without displacement of the trapping potentials; (b) bosons hosting a vortex line without displacement of the trapping potentials; (c) vortex-free configurations with a $10-\mu \mathrm{m}$ displacement between the trapping potentials; (d) bosons hosting a vortex line, plus a 10$\mu \mathrm{m}$ displacement between the trapping potentials.

We display in Fig. 3 the stability diagram for the ${ }^{40} \mathrm{~K}-{ }^{87} \mathrm{Rb}$ mixture in the $N_{B}-N_{F}$ plane. The dots are the theoretical prediction for $\left(N_{B}^{c}, N_{F}^{c}\right)$. The lines have been drawn to guide the eye and represent the critical instability lines that determine the boundary between the stable (left) and unstable (right) regions in four different cases: (a) vortexfree configurations in concentric trapping potentials (no displacement of the external fermion and boson potentials); (b) Bose condensate hosting a vortex line with bosons and fermions confined by concentric trapping potentials; (c) vortexfree configurations with a $10-\mu \mathrm{m}$ displacement between the trapping potentials; (d) Bose condensate hosting a vortex line, plus a $10-\mu \mathrm{m}$ displacement between the trapping potentials.

From Fig. 3 one can conclude that, for the ${ }^{40} \mathrm{~K}-{ }^{87} \mathrm{Rb}$ mixture, the presence of a vortex in the condensate, or any other mechanism that increases the distance between the maxima of the-still-overlapping densities, as for example a sag displacement between the two clouds, allows to have a stable mixture for larger particle numbers. The reason is that these mechanisms diminish the enhancement of the density of both species in the overlap volume caused by their attractive mutual interaction. A sag displacement (c) leads to critical numbers higher than the presence of a vortex (b), and when both are simultaneously present (d), they yield the larger stability region.

\section{SUMMARY}

We have studied Fermi-Bose mixtures with attractive mutual interaction, We have considered two potentially interest- 
ing cases. In one of them, bosons are in a vortex state, and in the other the minima of the trapping potentials for bosons and fermions are shifted from each other.

The position of the maximum of the fermion density is relevant for the collapse of the trapped boson-fermion mixture. Indeed, it is around these "fixed" points that the densities increase when the atom numbers approach their critical values, i.e., these are the points where the collapse starts. For this reason, simple analytical formulas have been derived to describe the effective potential felt by a small fermion amount in the presence of a large boson condensate with positive scattering length. These formulas have been used to seek the critical points of the fermion density distribution removing the restriction $N_{F} \ll N_{B}$. The positions of the critical points only depend on the value of a dimensionless parameter $\left(\gamma_{i}\right)$ we have introduced in Sec. III A, whose definition involves the values of the boson and fermion scattering lengths, masses, and confining frequencies.

The validity of these formulas has been assessed comparing the results obtained using them with the results obtained from the numerical solution of the mean-field equations (GP equation for bosons coupled to the TFW equation for fermions). We have numerically shown that the position of the maximum of the Fermionic density is very insensitive to the dilution value $N_{F} / N_{B}$, and have also checked that the analytical values obtained for a low number of fermions remain valid up to the critical atomic numbers.

Finally, we have shown that the critical atomic numbers that the mixture can sustain before it collapses may be increased shifting the-still overlapping — confining potentials, or when the condensate hosts a quantized vortex line. In the latter case, a larger overlap between both species has been found, which may favor sympathetic cooling.

\section{ACKNOWLEDGMENTS}

This work has been performed under Grant No. BFM2002-01868 from DGI, Spain, and Grant No. 2001SGR-00064 from Generalitat de Catalunya. D.M.J. has been also funded by the MECD (Spain) on sabbatical leave. M.G. thanks the "Ramón y Cajal" Program of the Ministerio de Ciencia y Tecnología (Spain) for financial support.

\section{APPENDIX}

In this appendix we use a scaling transformation $[14,20,21]$ to derive an expression that generalizes the virial theorem and is useful to test the accuracy of the numerical procedure. Performing a scaling transformation of the vector position $\vec{r} \rightarrow \lambda \vec{r}$, it is easy to check that to keep the normatization of the order parameter $\Psi(\vec{r})$ and of the boson and fermion atomic densities, they have to transform as

$$
\begin{gathered}
\Psi(\vec{r}) \rightarrow \Psi_{\lambda}(\vec{r})=\lambda^{3 / 2} \Psi(\lambda \vec{r}), \\
n(\vec{r}) \rightarrow n_{\lambda}(\vec{r})=\lambda^{3} n(\lambda \vec{r}) .
\end{gathered}
$$

Splitting the energy of the system into a kinetic $T$, a harmonic oscillator $U_{H}$, and an interaction part $U_{g}$, the above transformations induce the following ones:

$$
\begin{gathered}
T \rightarrow T_{\lambda}=\frac{\hbar^{2}}{2 M_{B}} \int d \vec{r}\left|\nabla \Psi_{\lambda}(\vec{r})\right|^{2}=\lambda^{2} T, \\
U_{H} \rightarrow U_{H_{\lambda}}=\frac{1}{2} M \omega^{2} \int d \vec{r} r^{2} n_{\lambda}=\frac{1}{\lambda^{2}} U_{H}, \\
U_{g} \rightarrow U_{g_{\lambda}}=\frac{1}{2} g \int d \vec{r} n_{\lambda}^{2}=\lambda^{3} U_{g},
\end{gathered}
$$

where the last two expressions hold for fermions and bosons as well. The expression for $U_{H}$ supposes a spherically symmetric harmonic trap, the generatization to deformed harmonic traps is obvious.

It is easy to check that in the case of fermions, the kinetic energy in the TFW approximation also scales as $T \rightarrow T_{\lambda}$ $=\lambda^{2} T$, and that in the case of boson-fermion mixtures, the interaction term $U_{g B F}$ scales as $U_{g B F} \rightarrow U_{g B F_{\lambda}}=\lambda^{3} U_{g B F}$. Hence the total energy of the mixture scales as

$$
\begin{aligned}
E \rightarrow & E_{\lambda}=\lambda^{2}\left(T_{B}+T_{F}\right)+\frac{1}{\lambda^{2}}\left(U_{H_{B}}+U_{H_{F}}\right)+\lambda^{3}\left(U_{g_{B B}}+U_{g_{F F}}\right. \\
& \left.+U_{g_{B F}}\right) .
\end{aligned}
$$

If the scaling is carried out from the equilibrium configuration corresponding to the value $\lambda=1$, one has

$$
\begin{aligned}
\left.\frac{d E_{\lambda}}{d \lambda}\right|_{\lambda=1}= & 2\left(T_{B}+T_{F}\right)-2\left(U_{H_{B}}+U_{H_{F}}\right)+3\left(U_{g_{B B}}+U_{g_{F F}}\right. \\
& \left.+U_{g_{B F}}\right)=0 .
\end{aligned}
$$

One sees that at equilibrium, for noninteracting fermions and bosons one recovers the virial theorem, namely $T_{B}=U_{H_{B}}$ and $T_{F}=U_{H_{F}}$.

When a vortex is present, its superfluid kinetic energy

$$
T_{V}=\frac{\hbar^{2}}{2 M_{B}} \int d \vec{r} \frac{n_{B}(\vec{r})}{r_{\perp}^{2}}
$$

scales as

$$
T_{V} \rightarrow T_{V_{\lambda}}=\frac{\hbar^{2}}{2 M_{B}} \int d \vec{r} \frac{n_{\lambda}(\vec{r})}{r_{\perp}^{2}}=\lambda^{2} T_{V}
$$

This is quite a natural result in view of the first Eq. (A2) and, as a consequence, $T_{V}$ may be incorporated in the definition of $T_{B}$ which then represents the total kinetic energy of the Bosonic component of the mixture.

If a gravitational sag is considered that displaces the atomic clouds in the $z$ direction, its effect can be taken into account changing the confining potential in that direction into $V(z)=\frac{1}{2} M \omega^{2}\left(z-z_{0}\right)^{2}$. Apart from a trivial constant term proportional to the number of fermions and bosons in the trap that is invariant under the scaling transformation, there appear new contributions to the total energy of the kind

$$
U_{s}=-M z_{0} \omega^{2} \int d \vec{r} z n(\vec{r})
$$

which scales as $U_{s} \rightarrow U_{s_{\lambda}}=\frac{1}{\lambda} U_{s}$. Equation (A4) then becomes 


$$
\begin{aligned}
\left.\frac{d E_{\lambda}}{d \lambda}\right|_{\lambda=1}= & 2\left(T_{B}+T_{F}\right)-2\left(U_{H_{B}}+U_{H_{F}}\right)-\left(U_{s_{B}}+U_{s_{F}}\right) \\
& +3\left(U_{g_{B B}}+U_{g_{F F}}+U_{g_{B F}}\right)=0 .
\end{aligned}
$$

The above expression is, in a way, a generalization of the virial theorem. We have used it to routinely check the accuracy in the numerical solution of the GP and TFW coupled equations. Values $\sim O(10)$ are found for that expression when $U_{H}$ is $\sim O\left(10^{6}\right)$. This makes us confident on the numerical method we have used to find the mean-field structure of the atomic mixture.
[1] A. G. Truscott, K. E. Strecker, W. I. McAlexander, G. B. Partridge, and R. G. Hulet, Science 291, 2570 (2001).

[2] F. Schreck, L. Khaykovich, K. L. Corwin, G. Ferrari, T. Bourdel, J. Cubizolles, and C. Salomon, Phys. Rev. Lett. 87, 080403 (2001).

[3] R. Roth, Phys. Rev. A 66, 013614 (2002).

[4] Z. Akdeniz, P. Vignolo, A. Minguzzi, and M. P. Tosi, J. Phys. B 35, L105 (2002).

[5] G. Ferrari, M. Inguscio, W. Jastrzebski, G. Modugno, and G. Roati, Phys. Rev. Lett. 89, 053202 (2002).

[6] G. Roati, F. Riboli, G. Modugno, and M. Inguscio, Phys. Rev. Lett. 89, 150403 (2002).

[7] M. Modugno, F. Ferlaino, F. Riboli, G. Roati, G. Modugno, and M. Inguscio, Phys. Rev. A 68, 043626 (2003).

[8] K. Molmer, Phys. Rev. Lett. 80, 1804 (1998).

[9] L. Vichi, M. Amoruso, A. Minguzzi, S. Stringari, and M. Tosi, Eur. Phys. J. D 11, 335 (2000).

[10] R. J. Donnelly, Quantized Vortices in Helium II (Cambridge University Press, Cambridge, England, 1991).

[11] M. R. Matthews, B. P. Anderson, P. C. Haljan, D. S. Hall, C. E. Wieman, and E. A. Cornell, Phys. Rev. Lett. 83, 2498 (1999).
[12] A. L. Fetter and A. A. Svidzinsky, J. Phys.: Condens. Matter 13, R135 (2001).

[13] C. J. Pethick and H. Smith, Bose-Einstein Condensation in Dilute Gases (Cambridge University Press, Cambridge, England, 2002).

[14] L. Pitaevskii and S. Stringari, Bose-Einstein Condensation (Clarendon Press, Oxford, 2003).

[15] R. Mayol, M. Pi, M. Barranco, and F. Dalfovo, Phys. Rev. Lett. 87, 145301 (2001).

[16] P. Capuzzi, A. Minguzzi, and M. P. Tosi, Phys. Rev. A 68, 033605 (2003).

[17] M. Pi, X. Viñas, F. Garcias, and M. Barranco, Phys. Lett. B 215, 5 (1988).

[18] M. Barranco, M. Guilleumas, E. S. Hernández, R. Mayol, M. Pi, and L. Szybisz, Phys. Rev. B 68, 024515 (2003).

[19] P. Capuzzi and E. S. Hernández, Phys. Rev. A 64, 043607 (2001).

[20] O. Bohigas, A. M. Lane, and J. Martorell, Phys. Rep. 51, 267 (1979).

[21] F. Dalfovo and S. Stringari, Phys. Rev. A 53, 2477 (1996). 